\title{
AN EQUIVALENCE THEOREM ON GENERATING FUNCTIONS ${ }^{1}$
}

\author{
H. M. SRIVASTAVA
}

ABSTRACT. The present paper establishes an equivalence theorem on generating relations for a sequence of functions $\left\{f_{n}(z)\right\}$ defined by the Rodrigues formula (1) below. It is also shown how this theorem may be applied to a fairly large variety of special functions including, for instance, the classical orthogonal polynomials.

1. The main result. We prove the following generalization of certain results on generating functions due to F. Brafman [1]:

Theorem. Let the function $F(z)$ be holomorphic on a domain $D$ of the complex z-plane, and define

$$
f_{n}(z)=(n !)^{-1} D_{z}^{n}\left\{(a z+b)^{n} F(z)\right\}, \quad D_{z}=d / d z,
$$

for $n=0,1,2, \ldots$, where $a$ and $b$ are complex constants, not both zero. Also let $\left\{\lambda_{n}\right\}$ be any sequence of complex numbers for which

$$
1 / R=\lim _{n \rightarrow \infty} \sup _{n}\left|\lambda_{n}\right|^{1 / n}
$$

is finite, or for which

$$
R=\lim _{n \rightarrow \infty}\left|\lambda_{n} / \lambda_{n+1}\right|
$$

exists and is positive. Suppose further that

$$
A_{n}=\sum_{k=0}^{[n / N]}\left(\begin{array}{c}
n \\
N k
\end{array}\right) \lambda_{k} w^{k}, \quad n=0,1,2, \ldots,
$$

for some positive integer $N$ and some complex number $w$.

Then

Received by the editors May 8, 1974 and, in revised form, August 15, 1974. AMS (MOS) subject classifications (1970). Primary 33A65, 33A45; Secondary 42A52, 30A86.

Key words and phrases. Equivalence theorem, generating relations, Rodrigues' formula, special functions, classical orthogonal polynomials, holomorphic functions, the Cauchy-Hadamard theorem, d'Alembert's ratio test, Cauchy's integral formula, uniform convergence, binomial theorem, analytic continuation, Hermite polynomials, Laguerre polynomials, Jacobi polynomials, Bessel polynomials, ultraspherical polynomials, Legendre polynomials, Tchebycheff polynomials, Charlier polynomials, generating-function equivalence, Meixner polynomials.

1 This work was supported in part by the National Research Council of Canada under grant A-7353. 


$$
\sum_{n=0}^{\infty} A_{n} f_{n}(z) t^{n}=\frac{1}{1-a t} \sum_{k=0}^{\infty} \lambda_{k} f_{N k}\left(\frac{z+b t}{1-a t}\right)\left[\frac{w t^{N}}{(1-a t)^{N}}\right]^{k},
$$

for some domain in the complex t-plane that includes the origin.

Proof. By the Cauchy-Hadamard theorem, $R$ in (2) is the radius of convergence of the series

$$
\sum_{n=0}^{\infty} \lambda_{n} Z^{n}
$$

By the d'Alembert ratio test, $R$ is also given by (3) in case that limit exists.

Note that

$$
\left|\frac{t}{1-a t}\right|<\rho \text { whenever }|t|<T(\rho)=\frac{\rho}{\rho|a|+1} .
$$

Let $z$ be any point in $D$, and let $C$ be a circle of radius $r$ (with centre at $z$ ) lying entirely within $D$. If we define

$$
z^{\prime}=(z+b t) /(1-a t)
$$

so that

$$
z^{\prime}-z=(a z+b) t /(1-a t)
$$

then, in view of (7), $z^{\prime}$ is within a distance $r / 2$ of $z$ whenever

$$
|t|<T(r / 2|a z+b|)
$$

By definition (1) and Cauchy's integral formula, we have

$$
f_{m}\left(z^{\prime}\right)=\frac{1}{2 \pi i} \int_{C} F(\zeta) \frac{(a \zeta+b)^{m}}{\left(\zeta-z^{\prime}\right)^{m+1}} d \zeta, \quad m=0,1,2, \ldots,
$$

whence the right-hand side of (5) may be written

$$
\Omega=\frac{1}{1-a t} \sum_{k=0}^{\infty} \lambda_{k} \frac{1}{2 \pi i} \int_{C} F(\zeta) \frac{(a \zeta+b)^{N k}}{\left(\zeta-z^{\prime}\right)^{N k+1}} d \zeta\left(\frac{w t^{N}}{(1-a t)^{N}}\right)^{k}
$$

Now consider the series

$$
S(\zeta)=\sum_{k=0}^{\infty} \lambda_{k} w^{k}\left(\frac{a \zeta+b}{\zeta-z^{\prime}} \frac{t}{1-a t}\right)^{N k}
$$

when $\zeta$ is on $C$. Since $\left|z^{\prime}-z\right|<r / 2$, we have $\left|\zeta-z^{\prime}\right|>r / 2$. Suppose $|a \zeta+b|<K$ when $\zeta$ is on $C$. Suppose further that 


$$
|t|<T\left(\left[\frac{R}{|w|}\right]^{1 / N} \frac{r}{2 K}\right)
$$

Then, by (7),

$$
\left|\frac{t}{1-a t}\right|<\left[\frac{R}{|w|}\right]^{1 / N} \frac{r}{2 K}
$$

The refore

$$
\left|\frac{a \zeta+b}{\zeta-z^{\prime}} \frac{t}{1-a t}\right|<\left[\frac{R}{|w|}\right]^{1 / N}
$$

It follows that series (12) converges uniformly in $\zeta$ when (3) holds.

This evidently justifies the inversion of the order of summation and integration in (11), and we thus have

$$
\Omega=\frac{1}{1-a t} \frac{1}{2 \pi i} \int_{C} F(\zeta) S(\zeta) \frac{d \zeta}{\zeta-z^{\prime}} .
$$

By (8) and (12), for $\zeta$ on $C$, we get

$$
\Delta=\frac{S(\zeta)}{(1-a t)\left(\zeta-z^{\prime}\right)}=\frac{1}{\zeta-z} \sum_{k=0}^{\infty} \lambda_{k} w^{k} \frac{W^{N k}}{(1-W)^{N k+1}},
$$

where, for convenience,

$$
W=(a \zeta+b) t /(\zeta-z)
$$

If

$$
|t|<r / K
$$

then $|W|<1$, and the denominator in series (15) can be expanded by means of the binomial theorem, giving

$$
\begin{aligned}
\Delta & =\frac{1}{\zeta-z} \sum_{k=0}^{\infty} \lambda_{k} w^{k} \sum_{m=0}^{\infty}\left(\begin{array}{c}
m+N k \\
N k
\end{array}\right) W^{m+N k} \\
& =\frac{1}{\zeta-z} \sum_{k=0}^{\infty} \lambda_{k} w^{k} \sum_{n=N k}^{\infty}\left(\begin{array}{c}
n \\
N k
\end{array}\right) W^{n} .
\end{aligned}
$$

To justify the interchange of the order of summation in this double series, we require that, in addition to (17), the infinite series in (15) converge if $\lambda_{k}, w$ and $W$ are replaced by $\left|\lambda_{k}\right|,|w|$ and $|W|$, respectively. Indeed, this modified series converges if $|w||W|^{N} /(1-|W|)^{N}<R$, that is, if $|W|<\left((|w| / R)^{1 / N}+1\right)^{-1}$. By $(16)$, this last inequality is satisfied if 


$$
|t|<r / K\left\{(|w| / R)^{1 / N}+1\right\} \text {. }
$$

Thus, if (17) and (19) hold, by interchanging the order of summation in (18) we obtain

$$
\Delta=\frac{1}{\zeta-z} \sum_{n=0}^{\infty} A_{n} W^{n},
$$

where $A_{n}$ and $W$ are given by (4) and (16), respectively.

Since the series on the right-hand side of (20) is uniformly convergent for $\zeta$ on $C$, therefore on substituting it into (14) we may interchange the order of summation and integration to get

$$
\Omega=\sum_{n=0}^{\infty} A_{n} \frac{1}{2 \pi i} \int_{C} F(\zeta) W^{n} \frac{d \zeta}{\zeta-z}=\sum_{n=0}^{\infty} A_{n} f_{n}(z) t^{n},
$$

by virtue of (16) and (10).

This last equation, together with (11), gives us (5), provided $|t|$ is constrained by (9), (13), (17), and (19), that is, $t$ lies in some neighbourhood of the origin. Evidently formula (5) may then be extended by analytic continuation on $t$ as far as possible.

2. Applications to classical orthogonal polynomials. In this section we consider several familiar instances of special functions each of which satisfies a Rodrigues formula of type (1). Indeed, in the notations used by Szegö [4] and others (cf., e.g., [2, Chapter X]), we have

$$
\begin{gathered}
f_{n}(z)=(n !)^{-1}(-1)^{n} \exp \left(-z^{2}\right) H_{n}(z)=(n !)^{-1} D_{z}^{n}\left\{\exp \left(-z^{2}\right)\right\}, \\
f_{n}(z)=z^{\alpha} e^{-z} L_{n}^{(\alpha)}(z)=(n !)^{-1} D_{z}^{n}\left\{z^{n+a} e^{-z}\right\}, \\
f_{n}(z)=z^{a-n} e^{-z} L_{n}^{(a-n)}(z)=(n !)^{-1} D_{z}^{n}\left\{z^{\alpha} e^{-z}\right\},
\end{gathered}
$$

$f_{n}(z)=2^{n}(z-1)^{\alpha}(z+1)^{\beta-n} P_{n}^{(\dot{\alpha}, \beta-n)}(z)=(n !)^{-1} D_{z}^{n}\left\{(z-1)^{n+\alpha}(z+1)^{\beta}\right\}$

$$
f_{n}(z)=2^{n}(z-1)^{\alpha-n}(z+1)^{\beta} P_{n}^{(\alpha-n, \beta)}(z)
$$

$$
\begin{gathered}
=(n !)^{-1} D_{z}^{n}\left\{(z+1)^{n+\beta}(z-1)^{\alpha}\right\}, \\
f_{n}(z)=2^{n}(z-1)^{\alpha-n}(z+1)^{\beta-n} P_{n}^{(\alpha-n, \beta-n)}(z) \\
=(n !)^{-1} D_{z}^{n}\left\{(z-1)^{\alpha}(z+1)^{\beta}\right\},
\end{gathered}
$$

for the classical orthogonal polynomials of Hermite, Laguerre, and Jacobi.

On the other hand, for the Bessel polynomials $y_{n}(z, \alpha-n, \beta)$ it is known 
that $[3$, p. 111, Equation (47)]

$$
f_{n}(z)=\frac{\beta^{n} z^{\alpha-n-2} e^{-\beta / z}}{n !} y_{n}(z, \alpha-n, \beta)=\frac{1}{n !} D_{z}^{n}\left\{z^{n+\alpha-2} e^{-\beta / z}\right\} .
$$

The result of the preceding section would apply also to the ultraspherical polynomials $P_{n}^{a}(z)$, the Legendre polynomials $P_{n}(z)$, and the Tchebycheff polynomials $U_{n}(z)$ of the second kind, since

$$
f_{n}(z)=(-1)^{n}\left(z^{2}-1\right)^{-a-n / 2} P_{n}^{\alpha}\left(\frac{z}{\sqrt{ }\left(z^{2}-1\right)}\right):=\frac{1}{n !} D_{z}^{n}\left\{\left(z^{2}-1\right)^{-a}\right\}
$$

and

$$
P_{n}(z)=P_{n}^{1 / 2}(z), \quad U_{n}(z)=P_{n}^{1}(z)
$$

Applying (5) to the special functions involved in equations (21) through (28) above, we shall be led to the following generating-function equivalences.

$$
\sum_{n=0}^{\infty} A_{n} H_{n}(z) \frac{t^{n}}{n !}=\exp \left(2 z t-t^{2}\right) \sum_{k=0}^{\infty} \lambda_{k} H_{N k}(z-t) \frac{\left(w t^{N}\right)^{k}}{(N k) !} .
$$

$$
\sum_{n=0}^{\infty} A_{n} L_{n}^{(\alpha)}(z) t^{n}=(1-t)^{-1-a} e^{-z t /(1-t)}
$$

$$
\begin{aligned}
& \sum_{n=0}^{\infty} A_{n} L_{n}^{(\alpha-n)}(z) t^{n}=(1+t)^{a} e^{-z t} \sum_{k=0}^{\infty} \lambda_{k} L_{N k}^{(\alpha-N k)}(z(1+t))\left(\frac{w t^{N}}{(1+t)^{N}}\right)^{k} . \\
& \sum_{n=0}^{\infty} A_{n} P_{n}^{(\alpha, \beta-n)}(z) t^{n}=(1-t)^{\beta}\{1-1 / 2(z+1) t\}^{-1-\alpha-\beta}
\end{aligned}
$$

$$
\text { - } \sum_{k=0}^{\infty} \lambda_{k} P_{N k}^{(\alpha, \beta-N k)}\left(\frac{z-(z+1) t / 2}{1-(z+1) t / 2}\right)\left(\frac{w t^{N}}{(1-t)^{N}}\right)^{k} \text {. }
$$$$
\sum_{n=0}^{\infty} A_{n} P_{n}^{(\alpha-n, \beta)}(z) t^{n}=(1+t)^{\alpha}\{1-1 / 2(z-1) t\}^{-1-\alpha-\beta}
$$

$$
\text { - } \sum_{k=0}^{\infty} \lambda_{k} P_{N k}^{(\alpha-N k, \beta)}\left(\frac{z+(z-1) t / 2}{1-(z-1) t / 2}\right)\left(\frac{w t^{N}}{(1+t)^{N}}\right)^{k}
$$


$\sum_{n=0}^{\infty} A_{n} P_{n}^{(a-n, \beta-n)}(z) t^{n}=\{1+1 / 2(z+1) t\}^{a}\{1+1 / 2(z-1) t\}^{\beta}$

$$
\begin{gathered}
\cdot \sum_{k=0}^{\infty} \lambda_{k} P_{N k}^{(a-N k, \beta-N k)}\left(z+1 / 2\left(z^{2}-1\right) t\right)\left(\frac{w t^{N}}{\{1+(z+1) t / 2\}^{N}\{1+(z-1) t / 2\}^{N}}\right)^{k} . \\
\sum_{n=0}^{\infty} A_{n} y_{n}(z, \alpha-n, \beta) \frac{t^{n}}{n !}=(1-z t / \beta)^{1-\alpha} e^{t} \\
\cdot \sum_{k=0}^{\infty} \lambda_{k} y_{N k}\left(\frac{z}{1-z t / \beta}, \alpha-N k, \beta\right) \frac{\left(w t^{N}\right)^{k}}{(N k) !} . \\
\sum_{n=0}^{\infty} A_{n} P_{n}^{a}(z) t^{n}=\mu^{-2 a} \sum_{k=0}^{\infty} \lambda_{k} P_{N k}^{a}\left(\frac{z-t}{\mu}\right)\left(w(t / \mu)^{N}\right)^{k},
\end{gathered}
$$

where, for convenience,

$$
\mu=\sqrt{ }\left(1-2 z t+t^{2}\right)
$$

\section{Concluding remarks.}

Remark 1. Since [4, p. 103]

$$
L_{n}^{(\alpha)}(z)=\lim _{\beta \rightarrow \infty} P_{n}^{(\alpha, \beta)}(1-2 z / \beta), \quad n=0,1,2, \ldots,
$$

the generating-function equivalence (32) may formally be derived as a limiting case of (34).

Remark 2. In view of the known relationship (cf., e.g., [2, p. 226, Equation (16)])

$$
L_{n}^{(\alpha-n)}(z)=(-z)^{n}(n !)^{-1} c_{n}(\alpha ; z),
$$

equation (32) can easily be restated in terms of the Charlier polynomials $c_{n}(x ; a)$ defined by [op. cit., Equation (4)]

$$
c_{n}(x ; \alpha)=\sum_{k=0}^{n}(-1)^{k}\left(\begin{array}{l}
n \\
k
\end{array}\right)\left(\begin{array}{l}
x \\
k
\end{array}\right) k ! \alpha^{-k}, \quad \alpha>0, \quad x=0,1,2, \ldots
$$

Remark 3. Results (33), (34) and (35) are essentially equivalent. Indeed, it is well known that [2, p. 169]

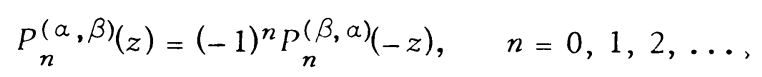

and that (cf. [4, p. 64]) 


$$
P_{n}^{(\alpha, \beta-n)}(z)=\left(\frac{1-z}{2}\right)_{n}^{n} P_{n}^{(-\alpha-\beta-n-1, \beta-n)}\left(\frac{z+3}{z-1}\right) .
$$

Remark 4. Since it is easily verified that

$$
y_{n}(z, \alpha-n, \beta)=n !(-z / \beta)^{n} L_{n}^{(1-\alpha-n)}(\beta / z),
$$

it is not difficult to deduce result (36) from (32), and vice versa.

Remark 5. By virtue of (29), the generating-function equivalence (37), with $\alpha=1 / 2$ and $\alpha=1$, will evidently yield the corresponding results for the Legendre polynomials and the Tchebycheff polynomials of the second kind.

Remark 6. An equivalence the orem for the Meixner polynomials $m_{n}(x ; \beta, c)$ would follow fairly readily from (33) by appealing to the known result $[2$, p. 226$]$

$$
m_{n}(x ; \beta, c)=n ! P_{n}^{(\beta-1,-\beta-n-x)}(2 / c-1) .
$$

The details may be omitted.

Finally, the author wishes to thank the referee for suggesting a number of improvements in this paper.

\section{REFERENCES}

1. F. Brafman, Generating functions and associated Legendre polynomials, Quart. J. Math. Oxford Ser. (2) 10 (1959), 156-160. MR $21 \# 6450$.

2. A. Erdélyi, W. Magnus, F. Oberhettinger and F. G. Tricomi, Higher transcendental functions. Vol. II, McGraw-Hill, New York, 1953. MR 15, 419.

3. H. L. Krall and O. Frink, A new class of orthogonal polynomials: The Bessel polynomials, Trans. Amer. Math. Soc. 65 (1949), 100-115. MR 10, 453.

4. G. Szegö, Orthogonal polynomials, Amer. Math. Soc. Colloq. Publ., vol. 23, Amer. Math. Soc., Providence, R. I., 1939. MR 1, 14.

DEP ARTMENT OF MATHEMATICS, UNIVERSITY OF VICTORIA, VICTORIA, BRITISH COLUMBIA, CANADA V8W 2 Y 2 